\title{
Psychoneuroimmunology: application to ocular diseases
}

\author{
Lori M. Ventura
}

Published online: 19 September 2009

(C) The Author(s) 2009. This article is published with open access at Springerlink.com

\section{Erratum to: $\mathrm{j}$ ocul biol dis inform DOI 10.1007/s12177-009-9028-4}

The following article was mistakenly run in volume 2 , issue 2 (June 2009) instead of in the present special issue, for which it was intended. The publisher regrets the error. Please also note additional author corrections as follows: on third page of the article, in the first paragraph under "Potential pathophysiological mechanisms," where was written cortisol release hormone (CRH)" now reads "Corticotrophin Releasing Hormone (CRH)." On the seventh page of the article, where was written " 25 -h" now reads " 24 -h."

\begin{abstract}
Psychoneuroimmunology (PNI) is a relatively new discipline within the field of neuroscience which researches the relationship between emotional states, the central and peripheral nervous systems, and the endocrine and immune systems. Negative psychological states, such as stress, anxiety, and depression, may alter immune system regulation and modulation of peripheral cytokines. A plethora of PNI studies have shown that increased psychological stress and depression are associated with an alteration of immune functioning and worsened health outcomes for many conditions. To date, application of PNI methodology has not been reported for ocular diseases. This article provides an historical perspective on the origins of the rift between the emotional and spiritual from physical aspects of disease. A review of how stress is mediated through sympathetic adrenomedullary and hypothalamic pituitary axis activation with shifts in
\end{abstract}

The online version of the original article can be found at http://dx.doi. org/10.1007/s12177-009-9028-4

NIH center grant P30-EY014801, unrestricted grant to Bascom Palmer Eye Institute from Research to Prevent Blindness.

L. M. Ventura $(\bowtie)$

University of Miami Miller School of Medicine,

Bascom Palmer Eye Institute,

Miami, FL, USA

e-mail: 1ventura@med.miami.edu immunity is provided. The literature which supports spirituality in healing is presented. Finally, ocular diseases which would be most amenable to a PNI approach are discussed.

Keywords Psychoneuroimmunology - Ocular disease . Spirituality · Stress · Emotions · Depression · Anxiety · Glaucoma $\cdot$ Macular degeneration $\cdot$ Central serous retinopathy $\cdot$ Keratitis sicca $\cdot$ Allergy

\section{The connection of mind, body, and spirit}

Many ancient cultures felt a connection between mind, body, and spirit. Illness has often been associated with sin $[1,2]$, and some societies sensed that healing could be restored by remaining in favor with the divine through an exorcism of evil spirits. The healing practice of shamanism is thought to have had its origins in Siberia dating as far back as 20,000 years ago. Shaman healers serve as mediaries between the spiritual and physical world. Once possibly universal, shamanism survives today primarily among indigenous peoples, and is especially widespread in Africa, Asia, and South America. As the practice of medicine evolved, the most insightful physicians clung to the belief that illness did not originate exclusively from that which is physical. Hippocrates in 400 B.C.E. stated: "I would rather know what sort of person has a disease than what sort of disease a person has." Ibn Sina, known by the Europeans as Avicenna, a Persian physician, philosopher, and scientist born in $980 \mathrm{CE}$, was fascinated by the effect of the mind on the body, and wrote a great deal on psychology. His most famous works, The Book of Healing and The Canon of Medicine, served as textbooks of medicine in Europe for the next 500 years. His feeling was that the evaluation of a "disease" was incomplete until and unless all components of a person's life had been included in the diagnosis.

Clinical medicine of the modern Western world has certainly eliminated the spiritual and, for the most part, the 
emotional from our healing practices. Patients delegate total authority to their doctors who must take on the magnanimous role as healer. Beinfield and Korngold [3] in their book Between Heaven and Earth wrote "As the breadth and depth of scientific information about the body grew, doctors and patients lost faith in the capacity of the human organism to heal itself. People mistakenly feel that the power to cure comes exclusively from outside themselves...administered by an alien intelligence." The doctor becomes the exclusive source of specialized knowledge, and is either the heroic slayer of disease, when their applications and surgical interventions have favorable outcomes, or are demonized for medication or surgical failures. Imbued as deity, physicians of today infrequently recognize a need for pastoral or psychiatry consults for our patients. A brief historical review explains how this schism occurred.

\section{The schism of mind, body, and spirit}

Until the Renaissance (1350), people were made to feel that they had to go outside themselves for salvation. The clergy were the sole interpreters of the scripture, the sole mediators between God and man, and the sole arbitrators of one's salvation.

By the 1500 s, a breach of faith caused people to become less dependent on the spiritual community of the church. During the Renaissance, there was a shift in focus from the spiritual to the acquisition of knowledge as religion was overshadowed by science, and the clergy lost their popularity to the mathematicians, the physicists, and the engineers. Science set itself out to explain everything including the nature of God, but when science could not explain God, a lack of security developed in Western culture, and man lost his spiritual footing. By the 17th century, Descartes declared, "There is nothing in the concept of body that belongs to the mind, and nothing in that of mind that belongs to the body." With this statement, Descartes chiseled a schism between the mind and the body, and dualism shattered the unity of mind, body, and spirit.

\section{Re-establishing the link of mind and body}

Despite this schism, a few insightful minds clung to the unity of ages past. Sir William Osler at the turn of the 20th century wrote, "The outcome of tuberculosis has more to do with the state of a patient's mind than the state of his lungs." It took 300 years for the seminal work of Ader and Cohen [4] to create a line of research known as psychoneuroimmunology which scientifically reestablished a link between emotions and disease.

Psychoneuroimmunology (PNI) is a relatively new discipline within the field of neuroscience which has its origins from the work of Ader and Cohen in the 1970s. PNI researches the relationship between emotional states, the central and peripheral nervous systems, and the endocrine and immune systems. Negative psychological states, such as stress, anxiety, and depression, may alter immune system regulation and modulation of peripheral cytokines. A plethora of PNI studies have shown that increased psychological stress and depression are associated with a decrease in immune functioning and worsened health outcomes for many conditions including coronary artery disease [5], cancer [6], autoimmune disease [7, 8], infection $[9,10]$, wound healing [11], and even Alzheimer's disease [12]. The literature is replete with examples for each of these conditions. For example, Kaprio et al. [13] showed that, in 95,647 widowed persons, the significant psychological stressor of loss of a spouse increases all-cause mortality. Breast cancer patients who are prone to stress have flattening of their diurnal cortisol slopes which has been associated with shorter survival times and greater progression [14]. The British Common Cold Study [15] showed that subjects with more stress had higher incidence of colds. Subsequently in 1998, the Pittsburgh Common Cold Study [16] showed that chronic interpersonal stressors such as conflicts with friends, family, or spouses and work problems related to unemployment or underemployment place individuals at greater risk for upper respiratory infection. Furthermore, there was a linear increase in the risk for illness with increasing duration of the stressor, and patients with greater perceived stress had worse cold symptoms and higher IL-6 levels. Poorer sleep efficiency and shorter sleep duration in the weeks preceding exposure to a rhinovirus were associated with lower resistance to illness [17].

From research on coping styles, it appears that the manner of responding to stressful events has implications both psychologically and physiologically [18]. Just as negative states have been cited to be associated with poorer outcomes, positive coping strategies have been correlated with more favorable health outcomes. For example, Carver [19] published that "finding benefit" among women with breast cancer during the year after diagnosis predicts better adjustment 5 to 8 years after diagnosis. Similarly in 2005, he published that an optimistic personality and psychosocial well-being during treatment predict psychosocial wellbeing among long-term survivors of breast cancer. A study by Penedo et al. [20] in men treated for localized prostate cancer suggests that optimism is associated with natural killer cell cytotoxicity and with a greater ability to adaptively express anger. In this group, it appears that less anger suppression partially mediates the relationship between optimism and natural killer cell cytotoxicity. Gonzalez et al. [21] conducted a cross-sectional study of HIV-positive men and women on highly active antiretroviral therapy, and 
showed that greater social support and positive states of mind related to better medication adherence, whereas higher depression scores related to non-adherence. The authors concluded that positive states of mind may be an important mechanism through which social support is related to better medication adherence in this population. Similarly, Ickovics et al. [22] showed that psychological resources in HIV-seropositive women, such as finding meaning in challenging circumstances and positive affect, may protect against HIV-related mortality and immune system decline.

Negative emotional states are as detrimental to the central nervous system as to the immune and other organ systems. Sheline et al. in 2003 showed that chronic depression leads to neuronal cell loss in the hippocampus [23]. Later, in 2006, Ownby et al. [12] concluded that a history of depression may confer an increased risk for later developing Alzheimer's disease, and that this relation may reflect an independent risk factor for the disease. If chronic depression leads to hippocampal volume loss, then negative emotional states may also be associated with deterioration of other areas of the central nervous system such as ocular structures. Patients who show onset or progression of ocular disease may acknowledge heightened emotional stress, yet among ophthalmologists, such a relationship has only been recognized for central serous retinopathy and reactivation of herpetic infection. There are three mechanisms by which negative emotional states or psychological stress may mediate injury to ocular structures, and potentially susceptible ophthalmic diseases are discussed.

\section{Potential pathophysiological mechanisms of psychological stress}

The first mechanism by which stress may cause injury to the eye is through diminished microvascular perfusion through sympathetic nervous system overdrive. Stressful stimuli may first be perceived visually or auditorily, or may be triggered by emotional signaling at the pre-frontal cortex. Whatever the origin of the stressor, there is signaling to the hippocampus for an interpretation based on memory. From the hippocampus, stimuli which evoke a fear response will synapse with neurons in the amygdala sending efferent projections to the paraventricular nucleus (PVN) of the hypothalamus which secretes corticotropinreleasing hormone (CRH) and arginine vasopressin (AVP) [11]. In addition to an antidiuretic effect, AVP increases peripheral vascular resistance to increase arterial blood pressure. Corticotropin-releasing hormone (CRH) travels in the hypothalamo-hypophyseal portal circulation to the pituitary (perhaps within $10 \mathrm{~s}$ ) to stimulate the release of $\mathrm{ACTH}$, enkephalins, and endorphins $[11,24]$. In addition to regulation of ACTH release, $\mathrm{CRH}$ flows diffusely throughout the brain, and serves as a neurotransmitter that mediates sympathetic arousal, providing an important link between the adrenocortical and autonomic branches of the stress response [24]. Noradrenergic centers in the brainstem (locus coeruleus) and spinal cord activate the sympathetic adrenomedullary (SAM) release of epinephrine from the adrenal medulla and norepinephrine from the peripheral autonomic nervous system. CRH and noradrenergic neurons in the CNS innervate and stimulate each other [11]. Acutely, the immediate response to psychological stress is activation of SAM, with vasoconstriction of blood vessels which may be narrowed to varying degrees from years of arteriolosclerosis.

Stress-induced vasospasm may compromise perfusion of the microvascular beds of the circulatory system, thereby inducing hypoxia, which may acutely exacerbate a number of ocular disease states including arteriolar obstructive phenomena, ischemic optic neuropathy, venous occlusive disease, and ischemic palsy of the cranial nerves. Initial ischemic insult with a sudden vaso-occlusive event or the subsequent reperfusion injury may induce damage. Chronic psychological stressors with ongoing activation of SAM result in vasoconstriction which may be prolonged, and may lead to hypertension and subsequent progression of arteriolosclerosis. Ocular conditions which may theoretically be exacerbated by chronic psychological stress would be any of the microvascular diseases such as hypertensive retinopathy, diabetic retinopathy, etc.

The second potential mechanism of how stress may exacerbate ocular disease is through the overdrive of the hypothalamic pituitary axis (HPA). The complexities of stressor-induced activation of the HPA and SAM (also known as sympathetic nervous system-SNS) have been comprehensively reviewed in other publications [25-27], and an abbreviated summary of the neural pathways provoked by stressors is provided here. Acutely stressful stimuli activate SAM as well as neurons of the hypothalamus to produce Corticotrophin Releasing Hormone (CRH). CRH-containing neurons stimulate the pituitary to release $\mathrm{ACTH}$, endorphins, and enkephalins into the bloodstream [11]. ACTH then stimulates the release of cortisol from the adrenal cortex. The HPA response to stress is not as immediate as SAM, but occurs within minutes [24]. Under normal non-stressed conditions, cortisol levels peak in the early morning hours, fall over the course of the day, in a fairly steep slope, to reach a low around 4PM, and remain low throughout the night only to peak again in the morning, and this is known as the diurnal fluctuation. Chronic psychological stress has been shown to elevate cortisol levels to induce a flattening of the diurnal cortisol slope [14]. Such chronic cortisol elevation is associated with worsening outcomes in breast [14] and ovarian cancer [28], and may have deleterious effects on ocular diseases 
(to be discussed later). There is an implied association between chronic stress and central serous chorioretinopathy (CSR), since CSR is known to occur in Cushing's disease with endogenous cortisol excess [29]. Ocular hypertension and glaucoma also occur in endogenous Cushing's disease [30].

The third potential mechanism of how stress may exacerbate ocular disease is through alteration of the immune profile. As mentioned, under psychologically stressful conditions, CRH and noradrenergic neurons in the CNS innervate and stimulate each other. Moynihan describes that activation of SAM and the HPA may have profound and complex downstream effects on immune function. Of the four categories of hormones that may change with stress (catecholamines, $\mathrm{CRH}$, endogenous opioids, and glucocorticoids), cortisol has received the greatest attention as the potential mediator of stress-induced changes in immunity [31]. The effects of stress on immunity are not unidirectional, and may vary based on three broad categories of variables: the experimental subject (age, sex, and strain), the nature of the stressor (intensity, duration, and perception), and the immune parameter examined (T helper 1 vs. T helper 2) [31]. For example, activation of the sympathetic nervous system primarily inhibits the activity of cells associated with the innate immune system, while it either enhances or inhibits the activity of cells associated with the acquired/adaptive immune system [32]. Whether the stressor is acute or chronic may alter the quality of the immune response dramatically. While acute stressors may enhance or suppress various facets of the immune response, chronic psychological stressors appear to be most often associated with suppressed immune function [31]. Chronic stress is associated with dysregulation in the negative feedback control of CRH-ACTH-cortisol loop resulting in chronic elevation of cortisol in the circulation [14].

Both aging and chronic stress may induce shifts in the immune system. Immune responses are regulated by antigen-presenting cells (APC) such as monocytes/macrophages, dendritic cells, microglia, and other phagocytic cells which are the components of innate immunity. Adaptive immune responses are mediated by $\mathrm{T}$ regulatory lymphocytes, T helper 1 cells (Th1) which promote cellular immunity, and $\mathrm{T}$ helper 2 cells (Th2) which enhance humoral immunity $[11,33]$. Th1 effects are mediated by cytokines interferon- $\gamma$, interleukin-2, interleukin-12, interleukin-18, TNF- $\alpha$, and T cells and macrophages. Th2 responses are mediated through the secretion of a different set of cytokines, primarily interleukin-1, interleukin-4, interleukin-6, interleukin-10, and interleukin-13, and B cells, eosinophils, and mast cells [11]. With aging, there is an involution of the thymus gland. Although not conclusive, most studies seem to suggest that aging is associated with a shift from a Th-1-dominated, cell-mediated immune response to a Th2 non-specific humoral response [33]. Chronic psychological stressors may induce a similar shift in immunity. Glucocorticoids and catecholamines mediate a Th2 shift by up-regulating Th2 cytokine production and also by suppressing APCs and Th1 cytokine synthesis [34, 35]. Through the above mechanisms, stress may influence the onset and/or course of infectious, autoimmune/inflammatory, allergic, and neoplastic diseases [35].

It should be noted that the nervous and immune systems are tightly connected. Tausk et al. [11] review the evidence for this as follows. First, CNS (hypothalamic) interventions (including lesioning studies) alter immunologic reactivity, and the elicitation of an immune response alters CNS activity. Second, there is sympathetic innervation of spleen and other lymphoid organs, including the skin [32]. Third, changes in hormone or transmitter levels produce changes in immune function and vice versa. Finally, lymphoid cells express receptors for a variety of hormones and transmitters [32]. Thus, lymphoid cells are exposed to neurochemical ligands, and express the receptors necessary to bind them. Tausk et al. further elaborate that the communication between the brain and immune system is bidirectional, with signaling back to the brain via the vagus nerve, and via soluble cytokines, including interleukin (IL)-6, IL-1, and tumor necrosis factor (TNF)- $\alpha[11,36,37]$.

\section{Diseases potentially susceptible to psychological stress}

\section{Glaucoma}

\section{Stress and vasospasm in glaucoma}

Retinal ganglion cells (RGCs) as one of the most metabolically active cells of the body have high numbers of mitochondria. Mitochondria play an important role in energy (ATP) production through the oxidative phosphorylation pathway and the regulation of cell death by apoptosis [38]. Mitochondria are particularly abundant along the unmyelinated intraretinal axons of the RGCs to supply energy in high demand for electrical conduction and axonal transport [39]. A steady supply of oxygen is necessary for oxidative phosphorylation to generate ATP. The microvascular supply of oxygen to RGC axons may deteriorate with aging as progressive arteriolosclerosis may result in vascular compromise of the inner retinal circulation. There is a natural loss of RGCs with aging in the normal population [40]. Several risk factors for glaucoma have been identified, some of which are strong including high intraocular pressure, increasing aging, family history of glaucoma, Black race, and other possible risk factors including high myopia, hypertension, diabetes, migraine 
headache, and vasospasm. These latter four implicate further vascular insult to an already compromised microvascular supply of oxygen secondary to the arteriolosclerotic changes of aging. Vasospasm secondary to psychological stress may further reduce perfusion in these diseased conditions, and theoretically worsen RGC and axonal function which may lead to premature apoptosis. Whether the ischemia itself and/ or reperfusion injury damages these structures is under investigation [38].

\section{Stress and hypothalamic-pituitary axis in glaucoma}

Endogenous elevation of cortisol levels secondary to chronic psychological stress may be damaging to the trabecular outflow apparatus depending on the concentration and duration of this elevation. Human trabecular meshwork cells contain glucocorticoid receptors [41, 42], and would therefore be expected to respond to glucocorticoid administration. The exogenous administration of glucocorticoids in man can generate a progressively elevated intraocular pressure (IOP) which is dependent on glucocorticoid potency, pharmacokinetics, duration of treatment, route of administration as well as differences in individual responsiveness [43]. Glucocorticoid-induced ocular hypertension is due to increased aqueous humor outflow resistance [43, 44]. Morphological examination of the trabecular meshwork of patients with glucocorticoidinduced glaucoma has shown an increased deposition of extracellular material in the trabecular beams and in the juxtacanalicular tissue, and a decrease in intratrabecular spaces [43, 45]. With topical, periocular, intravitreal, or oral administration of steroids for ocular inflammatory conditions, patients may suffer from a steroid-induced intraocular pressure elevation which may be severe and prolonged enough to require filtering surgery of the eye to lower the IOP. Jaffe et al. [46] studied the effects of intravitreal fluocinolone acetonide implants (Retisert) for treatment of uveitis. By week34 following implantation, $51.1 \%$ of implanted eyes required ocular antihypertensive drops, and 5.8\% underwent glaucoma filtering surgery. During this same period, the IOP of the fellow eyes did not rise significantly. In August of 2008, safety labeling changes were approved by FDA's Center for Drug Evaluation and Research (CDER). Based on clinical trials with Retisert, CDER warned that approximately $77 \%$ of patients will require IOP-lowering medications to control intraocular pressure and $37 \%$ of patients will require filtering procedures to control intraocular pressure within 3 years post implantation. Endogenous cortisol elevation of Cushing's disease is known to be associated with ocular hypertension [30]. At the Bascom Palmer Eye Institute, an anecdotal case of an intraocular pressure spike in a previously controlled glaucoma patient stricken by a severe chronic psychological stressor has been observed. Pressure elevation lasted for several weeks, and then returned to baseline levels as the patient coped with and adjusted to the stressor. The relationship between endogenous cortisol elevation and intraocular pressure responses requires further study.

\section{Immune balance in glaucoma}

Inflammatory mechanisms of glaucoma are being studied by different investigators. Michal Schwartz has pioneered the concept of harnessing the immune system to combat neurodegenerative diseases including glaucoma [47]. Her group has shown that immune deficiency or suppression impair the recovery process after optic nerve crush, whereas boosting a self-specific immune response, by both passive and active immunization, promotes recovery. Those same $\mathrm{T}$ cells that can lead to the development of autoimmune disease can protect neurons under neurodegenerative conditions, and it is a sub-population of regulatory $\mathrm{T}$ cells which regulates the autoimmune response to promote protection over injury. Dr. Schwartz' team introduced the concept of a therapeutic T-cell-mediated vaccination to boost the immune response to facilitate neuroprotection in glaucoma [48] .This immune defense involves lymphocytes, resident and infiltrating innate immune cells, the microglia, and macrophages. The antigens of choice are synthetic antigens, such as glatiramer acetate, that weakly cross-react with self-antigens in the retina and optic nerves. The vaccine induces a beneficial immune response that recruits immune effector cells to counteract or neutralize many of the compounds and factors that contribute to ongoing destruction, and in addition supports cell renewal and repair [49]. Working with Cohen et al. [50], Dr. Schwartz has also published that maladaptation to mental stress in mice was mitigated by the adaptive immune system via depletion of naturally occurring regulatory CD4+CD25+ cells. This begs the question of how maladaptation to psychological stress may affect immune balance in patients with glaucoma. A different team led by Wax and Tezel has proposed that the inflammatory cytokine TNF- $\alpha$ may be harmful to retinal ganglion cells thereby having an etiologic role in glaucoma [51, 52]. While ischemic insults are implicated as the trigger for TNF- $\alpha$-mediated damage to the RGC, it is not known what effect stress may have as an inciting factor.

When IOP is markedly elevated, clinicians often inquire about the recent use of oral, topical, or injected steroids. Clinicians may also consider inquiry regarding potential life or environmental stressors in the context of a previously well-controlled glaucoma patient who develops a dramatic IOP increase or sudden deterioration of function. 


\section{Keratitis sicca}

An optimal ocular surface depends on the healthy interplay of the immune system and neural circuitry. Inflammatory mechanisms in the pathogenesis of keratoconjunctivitis sicca have been well documented [53, 54]. The cornea is provided with the richest innervation of all body tissues, and it is generally accepted that corneal sensory innervation, via the ophthalmic division of the trigeminal nerve, plays a key role in maintaining anatomical integrity and function of the cornea and particularly of the epithelium [55]. Experimental and clinical evidence suggests a bidirectional control of corneal epithelium proliferation: sensory neuromediators promote epithelial cell mitosis, while, on the other hand, sympathetic mediators, epinephrine and norepinephrine, reduce epithelial cell mitosis [56]. In addition, in animal studies, corneal epithelial abnormalities induced by lesioning of the sensory nerves can be reduced by cervical sympathetic denervation $[55,57,58]$. Of similar interest is that Liu et al. recently published that all five subtypes of muscarinic cholinergic receptors are found on corneal and conjunctival cells, and these muscarinic receptors have a role in epithelial cell proliferation similar to that of epithelial growth factor (EGF) [59]. Parasympathetic innervation of the lacrimal gland sponsors proper secretory function, yet the relationship of dry eye to psychological stress-induced autonomic dysregulation and catecholamine excess has not been explored. In general, chronic stress and sympathetic overdrive is linked to parasympathetic inhibition. How parasympathetic impedance affects lacrimal gland stimulation, muscarinic receptors, and the ocular surface under conditions of psychological stress requires further study.

\section{Allergy, atopy, and uveitis}

Based on the shifts from Th1 to Th2 immunity discussed earlier, it is not surprising that clinicians may note progression or exacerbation of allergic conditions with aging and stress. The condition of atopic dermatitis exemplifies the delicate balance between genetic, environmental, and psychological factors in the evolution of the dermal and, quite possibly, the ocular expression of the disease. In a recent publication in Dermatologic Therapy, Tausk et al. [11] describes that patients with atopic dermatitis have an inherited deficiency in the HPA axis in that they respond with a blunted production of cortisol in response to experimental stressors. In addition, these patients display an up-regulation of glucocorticoid receptors on peripheral leukocytes, making them hyper-reactive to steroid stimulation. The authors posit that, despite a blunted HPA axis response to stress, effector cells that are exquisitely sensitive to systemic glucocorticoid release may respond in a hyper-reactive fashion to stress-induced cortisol elevations, accentuating the cytokine shift from the Th1 to the Th2 immune response. Pharmacologic doses of corticosteroids are also able to suppress the production of IL-4, which is a cytokine that mediates differentiation towards Th2 cells, explaining the beneficial effects of systemic or topical administration of these agents [11]. Ocular manifestations of atopic dermatitis in the form of keratoconjunctivitis may also be likely to be exacerbated under conditions of stress. Along similar lines, while no known cause can be identified in the vast majority of cases of acute anterior uveitis, clinicians who take a thorough social history may discover that there is a predisposing stressful event triggering the inflammatory response. Ophthalmologists and optometrists may inquire about potential psychological and environmental stressors when confronted with a patient in the throes of an acute ocular presentation of these conditions.

\section{Age-related macular degeneration}

Age-related macular degeneration (AMD) is the leading cause of legal blindness in persons age 65 years or older in the USA. The prevalence of AMD increases dramatically with age. A series of studies indicate the prevalence of dry AMD to be as high as $15 \%$ by age 65 increasing to as much as $30 \%$ by age 80 . Advanced AMD affects more than 1.75 million individuals in the USA [60]. Owing to the steady increase in life expectancy of the US population, this number is expected to increase by $50 \%$ to almost 3 million by 2020 . Recent evidence strongly implicates immune system activation of the alternative pathway of complement in the pathogenesis of AMD [61]. Dry AMD may convert to the wet form of advanced AMD, mediated by a cytokine called vascular endothelial growth factor (VEGF) [62]. Lutgendorf et al. showed that elevated levels of vascular endothelial growth factor were associated with lesser degrees of social support in women with ovarian cancer [63]. Lutgendorf's findings raise the question of whether similar imbalances may be at play in the immune-mediated setting of age-related macular degeneration, a disease of the elderly where a high incidence of anxiety has been reported [64]. The relationship between stress and AMD onset and progression is intriguing.

\section{Ocular infection}

A shift of Th1 to Th2 immunity with either aging or stress may enhance susceptibility to viral infection. Clinically, it is known that herpes simplex virus or herpes zoster virus reactivation may occur in the face of psychological or environmental stress. In addition, depression may have an effect on innate immunity. In HIV-infected women, a study by Evans et al. suggests that depression may decrease 
natural killer cell activity and lead to an increase in viral load [65]. In fact, a large epidemiology study found a significant association between depression and HIV morbidity and mortality in HIV-seropositive women. The mortality rate was doubled in the women with chronic depressive symptoms compared with women with limited or no depressive symptoms [66]. Such alteration of innate and adaptive immunity with negative psychological states may also be associated with ocular infections such as reactivation of ocular herpetic disease and toxoplasmosis, and adenoviral keratoconjunctivitis. The latter seems reasonable in view of the association of increased stress with viral upper respiratory infection published in the British Common Cold Study [15] and the Pittsburgh Common Cold Study [16] mentioned earlier.

\section{Ocular malignancy}

The shift away from Th1 or cellular mediated immunity with stress and aging may lead to onset of or progression of malignancy. Chronic stress has been shown to alter the diurnal cortisol curve with sustained elevation of cortisol levels throughout the day and night to induce a flattening of the diurnal cortisol slope. As shown by Sephton et al. [14], this flattening of the cortisol rhythmicity has been shown to predict shorter survival among women with metastatic breast cancer. Lutgendorf has shown a significant relationship between cortisol dysregulation, interleukin-6 (IL-6), and vegetative depression in women with ovarian cancer [28]. Parker et al. reported a model of stress-induced carcinogenicity where UV-treated mice repeatedly exposed to a predator scent developed squamous cell carcinomas significantly earlier compared to non-stressed controls [67, 68]. Such negative outcomes spawn the question of whether flattened cortisol curves (that is, chronically elevated cortisol) may pose an increased risk for poor outcomes in ocular malignancies such as melanoma and metastatic carcinoma, and periocular malignancies such as squamous cell, basal cell, sebaceous gland carcinoma, adenoid cystic carcinoma, etc. in predisposed individuals.

\section{Wound healing}

Kiecolt-Glaser and colleagues investigated the quality of marital relationships and the partners' time to heal a dermal blister wound. For those couples who engage in hostile interactions, the median time to wound healing was 2 days longer than for those couples who have non-hostile interactions. (7 vs. 5 days) [69]. A study in older adults, mean age 65 , showed that exercise intervention with $30 \mathrm{~min}$ of cardio exercise 3 days/week healed a 3.5 dermal wound significantly faster than participants in a non-exercise group (29.2 vs. 40 days, on average) [70]. Tausk draws the conclusion that the process of wound healing is affected by stressful life conditions, and states that this can be improved through intervention or changes in lifestyle. Before taking a patient to surgery, a line of questioning about stress may shed light on events in the patient's life which may compromise their surgical result. Loss of a loved one and divorce are particularly emotionally destabilizing, and are associated with greater morbidity [6] and mortality [13]. Postponement of the procedure to a more favorable time would be optimal to facilitate timely wound healing and prevent postoperative complications.

\section{Spirituality acknowledged}

While there is general agreement that religion and spirituality provide psychological comfort to patients, the physical health benefits of spirituality, religion, and prayer remain controversial due to a paucity of data from well-controlled, methodologically sound investigations. Ironson [1] cites major works which have reviewed hundreds of epidemiologic studies, and the conclusion was that there was probable validity to an association between religion and health, and that there may be a causal link. Patients may identify themselves as spiritual but not religious, thus the Ironson-Woods Spirituality/Religiousness Index is a measure that was developed to capture spirituality and religiosity, and the private and public expression of both. Evaluating four factors, a sense of peace, faith in God, religious behavior, and a compassionate view of others, Ironson et al. [1] used this measure to examine the relation between spirituality and religiousness and important health outcomes for people living with HIV, and to examine the potential mediators of these relations. Long survival in patients living with HIV was significantly related to the frequency of prayer (positively), and negatively related to a judgmental attitude. In addition, spirituality and religiousness yielded strong and significant correlations with less distress, more hope, social support, health behaviors, helping others, and lower cortisol levels. The relation between religious behavior and health outcomes was not due to social support. Upon further analysis, the authors identified urinary cortisol concentrations and altruistic behavior as mediators of the relation between spirituality, religiousness, and long survival. A later study by Carrico et al. [71] concluded that spirituality (using the IronsonWoods Spirituality/Religiousness Index) was associated with higher positive reappraisal coping and greater benefit finding, which, in turn, were both related to lower depressive symptoms in 264 HIV-positive men and women on highly active antiretroviral therapy. Furthermore, benefit finding in this group was uniquely predictive of decreased 24-h urinary free cortisol output indicating lower stress levels. 
Several studies have shown that spirituality and attendance of religious services has been shown to reduce mortality. A review of well-controlled studies that examined the relationship between religiosity and health reported the average reduction in risk of mortality in individuals who frequently attend religious services to be approximately $30 \%$; this drops to approximately $25 \%$ when adjusting for potential confounding variables [72]. Hummer et al. [73] found that weekly church/worship service attendance, compared with less or no attendance, protected against the onset of cardiovascular disease in a sample of 21,204 adults followed for 8 years. Other large studies [74] have confirmed this finding. On the other hand, in a much smaller cohort of only 503 patients, a study by Blumenthal et al. [74] found little evidence that self-reported spirituality, frequency of church attendance, or frequency of prayer is associated with cardiac morbidity or all-cause mortality after acute myocardial infarction in patients with depression and/or low perceived support. The authors mention that this study used a restricted sample of patients who were depressed and/or perceived themselves as having low social support, and this may explain their failure to corroborate the results of the other studies. Variations in population characteristics and in spiritual measures may underlie the controversy in published data relating spirituality to health outcomes and mortality.

In 2004, Lutgendorf et al. [75] provided support for the hypothesis that more frequent religious attendance in a population-based sample of older adults is associated with lower mortality and lower levels of interleukin-6 (IL-6), a cytokine linked to a number of age-related diseases. These findings were independent of potential confounding variables, such as age, sex, BMI, CRP, physical illness, social network size, depression, and history of cardiovascular disease, which were controlled in the structural equation models. IL-6 levels are known to increase with chronic stress and depression [76] and to decrease following successful treatment of depression [77]. IL-6 promotes angiogenesis in ovarian cancer and supports tumor growth and metastatic spread [78]. Similar well-controlled studies which explore the relationship of different facets of spirituality and religiousness to the underlying immune and neurobiological mechanisms of disease are necessary.

\section{Conclusion}

A multitude of studies over the years have attempted to establish a relationship between psychological stress and medical illness [33]. It is clear from the plethora of articles in all fields of medicine that no organ system is protected from the effects of negative emotional states. As a part of the central nervous system, and privy to the alarmed cross- talk of local hormones and neurotransmitters, one would surmise that the eye would be particularly vulnerable to the effects of psychological stress. It is surprising that vision researchers have not embraced the approach of psychoneuroimmunology in addressing ocular disease.

Evidence suggests stress has a higher prevalence among the elderly, and that anxiety disorders and phobias are more common in the aged than the general population [64]. The conditions which are most likely to induce chronic debilitating stress are the emotional adjustments of retirement, reduction in income, chronic illness, loss of mobility or function, isolation from or loss of loved ones, divorce, chronic strain such as caregiver's stress, problems with children, re-location, and many others. With increasing clinic loads and less time spent with each patient, ophthalmologists barely have enough time to conduct a proper past medical history or review of systems, thus a more intimate line of questioning regarding a patient's home life or emotional status is simply not undertaken. Certain ocular conditions, as mentioned above, require that physicians probe into the emotional status of their patients to expose the underpinnings of the disease process and to optimize the potential for recovery without recurrence.

Perhaps without realizing it, physicians minister the spirit with a cheerful disposition, through addressing and listening to their fears and concerns, with phrasing of optimistic perspectives, and by the mere touch of a caring hand upon a patient's shoulder. Thus, the spirituality of healing does not rest exclusively within the realm of the patient. In contrast, a rushed physician may miss the opportunity to establish this sacred and delicate relationship with the patient through failure to explain or reassure in a number of ways. During a busy clinic, this may often occur without the physician's awareness of having slighted the patient. Particularly in the field of ophthalmology where patients arrive for their visit frightened about the prospect of debilitating visual loss or blindness, the good doctor will tend not only their ocular condition but their emotional and spiritual perspective regarding their ailment. It is helpful to prioritize time with the patient to establish a compassionate rapport which facilitates dialogue to assess these factors in a gentle manner. Questions that address difficulty sleeping, problems at work, tension in the home, irritability, worry, and fear are necessary to know and understand a patient's inner struggle. Failure to do so compromises the individual's opportunity for healing. As research lends more support for the benefit of a spiritual approach to healing, we may tap into a valuable therapeutic resource.

Open Access This article is distributed under the terms of the Creative Commons Attribution Noncommercial License which permits any noncommercial use, distribution, and reproduction in any medium, provided the original author(s) and source are credited. 


\section{References}

1. Ironson G, et al. The Ironson-Woods Spirituality/Religiousness Index is associated with long survival, health behaviors, less distress, and low cortisol in people with HIV/AIDS. Ann Behav Med. 2002;24(1):34-48.

2. Kingsley D. Health, healing, and religion. A cross-cultural perspective. Upper Saddle River: Prentice Hall; 1996.

3. Beinfield H, Korngold E. Between heaven and earth: a guide to Chinese medicine. 1st Trade pbk. ed. New York: Ballantine; 1992. p. 432. xvi.

4. Ader R, Cohen N. Behaviorally conditioned immunosuppression. Psychosom Med. 1975;37(4):333-40.

5. Hemingway $\mathrm{H}$, Kuper H, Marmot M. Psychosocial factors in the primary and secondary prevention of coronary heart disease: an updated systematic review of prospective cohort studies. In: Yusuf S, Cairns JA, Camm AJ, Fallen EL, Gersh BJ, editors. Evidencebased cardiology. 2nd ed. London: BMJ; 2003. p. 181-218.

6. Lillberg K, et al. Stressful life events and risk of breast cancer in 10,808 women: a cohort study. Am J Epidemiol. 2003;157 (5):415-23.

7. Feigenbaum SL, Masi AT, Kaplan SB. Prognosis in rheumatoid arthritis. A longitudinal study of newly diagnosed younger adult patients. Am J Med. 1979;66(3):377-84.

8. Waltz M, Kriegel W, van't Pad Bosch P. The social environment and health in rheumatoid arthritis: marital quality predicts individual variability in pain severity. Arthritis Care Res. 1998;11(5):356-74.

9. Luborsky L, et al. Herpes simplex virus and moods: a longitudinal study. J Psychosom Res. 1976;20(6):543-8.

10. Friedman E, Katcher AH, Brightman VJ. Incidence of recurrent herpes labialis and upper respiratory infection: a prospective study of the influence of biologic, social and psychologic predictors. Oral Surg Oral Med Oral Pathol. 1977;43(6):873-8.

11. Tausk F, Elenkov I, Moynihan J. Psychoneuroimmunology. Dermatol Ther. 2008;21(1):22-31.

12. Ownby RL, et al. Depression and risk for Alzheimer disease: systematic review, meta-analysis, and metaregression analysis. Arch Gen Psychiatr. 2006;63(5):530-8.

13. Kaprio J, Koskenvuo M, Rita H. Mortality after bereavement: a prospective study of 95,647 widowed persons. Am J Public Health. 1987;77(3):283-7.

14. Sephton SE, et al. Diurnal cortisol rhythm as a predictor of breast cancer survival. J Natl Cancer Inst. 2000;92(12):994-1000.

15. Cohen S, Tyrrell DA, Smith AP. Psychological stress and susceptibility to the common cold. N Engl J Med. 1991;325 (9):606-12.

16. Cohen S, et al. Types of stressors that increase susceptibility to the common cold in healthy adults. Health Psychol. 1998;17(3):214-23.

17. Cohen S, et al. Sleep habits and susceptibility to the common cold. Arch Intern Med. 2009;169(1):62-7.

18. Spiegel D. Healing words: emotional expression and disease outcome. Jama. 1999;281(14):1328-9.

19. Carver CS, et al. Optimistic personality and psychosocial well-being during treatment predict psychosocial well-being among long-term survivors of breast cancer. Health Psychol. 2005;24(5):508-16.

20. Penedo FJ, et al. Anger suppression mediates the relationship between optimism and natural killer cell cytotoxicity in men treated for localized prostate cancer. J Psychosom Res. 2006;60(4):423-7.

21. Gonzalez JS, et al. Social support, positive states of mind, and HIV treatment adherence in men and women living with HIV/ AIDS. Health Psychol. 2004;23(4):413-8.

22. Ickovics JR, et al. Psychological resources protect health: 5-year survival and immune function among HIV-infected women from four US cities. Aids. 2006;20(14):1851-60.
23. Sheline YI, Gado MH, Kraemer HC. Untreated depression and hippocampal volume loss. Am J Psychiatry. 2003;160(8): 1516-8.

24. Sapolsky RM, Romero LM, Munck AU. How do glucocorticoids influence stress responses? Integrating permissive, suppressive, stimulatory, and preparative actions. Endocr Rev. 2000;21(1): $55-89$.

25. Chrousos GP. Stressors, stress, and neuroendocrine integration of the adaptive response. The 1997 Hans Selye Memorial Lecture. Ann N Y Acad Sci. 1998;851:311-35.

26. Charmandari E, Tsigos C, Chrousos G. Endocrinology of the stress response. Annu Rev Physiol. 2005;67:259-84.

27. McEwen BS. Protection and damage from acute and chronic stress: allostasis and allostatic overload and relevance to the pathophysiology of psychiatric disorders. Ann N Y Acad Sci. 2004;1032:1-7.

28. Lutgendorf SK, et al. Interleukin-6, cortisol, and depressive symptoms in ovarian cancer patients. J Clin Oncol. 2008;26 (29):4820-7.

29. Garg SP, et al. Endogenous cortisol profile in patients with central serous chorioretinopathy. Br J Ophthalmol. 1997;81(11):962-4.

30. Huschle OK, et al. Glaucoma in central hypothalamic-hypophyseal Cushing syndrome. Fortschr Ophthalmol. 1990;87 (5):453-6.

31. Moynihan JA. Mechanisms of stress-induced modulation of immunity. Brain Behav Immun. 2003;17(Suppl 1):S11-6.

32. Nance DM, Sanders VM. Autonomic innervation and regulation of the immune system (1987-2007). Brain Behav Immun. 2007;21(6):736-45.

33. Vedhara KaIM. Human psychoneuroimmunology. Oxford: Oxford University Press; 2005.

34. Blotta MH, DeKruyff RH, Umetsu DT. Corticosteroids inhibit IL12 production in human monocytes and enhance their capacity to induce IL-4 synthesis in CD4+ lymphocytes. J Immunol. 1997;158(12):5589-95.

35. Elenkov IJ, Chrousos GP. Stress, cytokine patterns and susceptibility to disease. Baillieres Best Pract Res Clin Endocrinol Metab. 1999;13(4):583-95.

36. Watkins LR, Maier SF, Goehler LE. Cytokine-to-brain communication: a review \& analysis of alternative mechanisms. Life Sci. 1995;57(11):1011-26.

37. Maier SF, et al. The role of the vagus nerve in cytokine-to-brain communication. Ann N Y Acad Sci. 1998;840:289-300.

38. Kong GY, et al. Mitochondrial dysfunction and glaucoma. J Glaucoma. 2009;18(2):93-100.

39. Wang L, et al. Varicosities of intraretinal ganglion cell axons in human and nonhuman primates. Invest Ophthalmol Vis Sci. 2003;44(1):2-9.

40. Porciatti V, Ventura LM. Normative data for a user-friendly paradigm for pattern electroretinogram recording. Ophthalmology. 2004;111(1):161-8.

41. Weinreb RN, et al. Detection of glucocorticoid receptors in cultured human trabecular cells. Invest Ophthalmol Vis Sci. 1981;21(3):403-7.

42. Hernandez MR, et al. Glucocorticoid target cells in human outflow pathway: autopsy and surgical specimens. Invest Ophthalmol Vis Sci. 1983;24(12):1612-6.

43. Wordinger RJ, Clark AF. Effects of glucocorticoids on the trabecular meshwork: towards a better understanding of glaucoma. Prog Retin Eye Res. 1999;18(5):629-67.

44. Bernstein HN, Schwartz B. Effects of long-term systemic steroids on ocular pressure and tonographic values. Arch Ophthalmol. 1962;68:742-53.

45. Rohen JW, Linner E, Witmer R. Electron microscopic studies on the trabecular meshwork in two cases of corticosteroid-glaucoma. Exp Eye Res. 1973;17(1):19-31. 
46. Jaffe GJ, et al. Fluocinolone acetonide implant (Retisert) for noninfectious posterior uveitis: thirty-four-week results of a multicenter randomized clinical study. Ophthalmology. 2006;113 (6):1020-7.

47. Schwartz M. Lessons for glaucoma from other neurodegenerative diseases: can one treatment suit them all? J Glaucoma. 2005;14 (4):321-3.

48. Bakalash $\mathrm{S}$, et al. T-cell-based vaccination for morphological and functional neuroprotection in a rat model of chronically elevated intraocular pressure. J Mol Med. 2005;83(11):904-16.

49. Schwartz M. Modulating the immune system: a vaccine for glaucoma? Can J Ophthalmol. 2007;42(3):439-41.

50. Cohen $\mathrm{H}$, et al. Maladaptation to mental stress mitigated by the adaptive immune system via depletion of naturally occurring regulatory $\mathrm{CD} 4+\mathrm{CD} 25+$ cells. J Neurobiol. 2006;66(6): 552-63.

51. Yan X, et al. Matrix metalloproteinases and tumor necrosis factor alpha in glaucomatous optic nerve head. Arch Ophthalmol. 2000;118(5):666-73.

52. Tezel G, Wax MB. Increased production of tumor necrosis factoralpha by glial cells exposed to simulated ischemia or elevated hydrostatic pressure induces apoptosis in cocultured retinal ganglion cells. J Neurosci. 2000;20(23):8693-700.

53. Pflugfelder SC, et al. Epithelial-immune cell interaction in dry eye. Cornea. 2008;27(Suppl 1):S9-11.

54. Lam H, et al. Tear cytokine profiles in dysfunctional tear syndrome. Am J Ophthalmol. 2009;147(2):198-205. e1.

55. Bonini S, et al. Neurotrophic keratitis. Eye. 2003;17(8):989-95.

56. Cavanagh HD, Colley AM. The molecular basis of neurotrophic keratitis. Acta Ophthalmol Suppl. 1989;192:115-34.

57. Fujita S, Miyazono Y, Ohba N. Capsaicin-induced corneal changes associated with sensory denervation in neonatal rat. Jpn J Ophthalmol. 1987;31(3):412-24.

58. Shimizu T, et al. Capsaicin-induced corneal lesions in mice and the effects of chemical sympathectomy. J Pharmacol Exp Ther. 1987;243(2):690-5.

59. Liu S, et al. Expression and function of muscarinic receptor subtypes on human cornea and conjunctiva. Invest Ophthalmol Vis Sci. 2007;48(7):2987-96.

60. Friedman DS, et al. Prevalence of age-related macular degeneration in the United States. Arch Ophthalmol. 2004;122(4):564-72.

61. Hageman GS, et al. An integrated hypothesis that considers drusen as biomarkers of immune-mediated processes at the RPEBruch's membrane interface in aging and age-related macular degeneration. Prog Retin Eye Res. 2001;20(6):705-32.
62. Lip PL, et al. Age-related macular degeneration is associated with increased vascular endothelial growth factor, hemorheology and endothelial dysfunction. Ophthalmology. 2001;108(4):705-10.

63. Lutgendorf SK, et al. Vascular endothelial growth factor and social support in patients with ovarian carcinoma. Cancer. 2002;95(4):808-15.

64. Kirmizioglu Y, et al. Prevalence of anxiety disorders among elderly people. Int J Geriatr Psychiatry, 2009;1099-1166 [Epub ahead of print].

65. Evans DL, et al. Association of depression with viral load, CD8 T lymphocytes, and natural killer cells in women with HIV infection. Am J Psychiatry. 2002;159(10):1752-9.

66. Ickovics JR, et al. Mortality, CD4 cell count decline, and depressive symptoms among HIV-seropositive women: longitudinal analysis from the HIV Epidemiology Research Study. Jama. 2001;285(11):1466-74.

67. Parker J, et al. Chronic stress accelerates ultraviolet-induced cutaneous carcinogenesis. J Am Acad Dermatol. 2004;51(6):919-22.

68. Saul AN, et al. Chronic stress and susceptibility to skin cancer. J Natl Cancer Inst. 2005;97(23):1760-7.

69. Kiecolt-Glaser JK, et al. Hostile marital interactions, proinflammatory cytokine production, and wound healing. Arch Gen Psychiatry. 2005;62(12):1377-84.

70. Emery CF, et al. Exercise accelerates wound healing among healthy older adults: a preliminary investigation. J Gerontol A Biol Sci Med Sci. 2005;60(11):1432-6.

71. Carrico AW, et al. A path model of the effects of spirituality on depressive symptoms and 24-h urinary-free cortisol in HIVpositive persons. J Psychosom Res. 2006;61(1):51-8.

72. Powell LH, Shahabi L, Thoresen CE. Religion and spirituality. Linkages to physical health. Am Psychol. 2003;58(1):36-52.

73. Hummer RA, et al. Religious involvement and U.S. adult mortality. Demography. 1999;36(2):273-85.

74. Blumenthal JA, et al. Spirituality, religion, and clinical outcomes in patients recovering from an acute myocardial infarction. Psychosom Med. 2007;69(6):501-8.

75. Lutgendorf SK, et al. Religious participation, interleukin-6, and mortality in older adults. Health Psychol. 2004;23(5):465-75.

76. Maes M, et al. Increased serum IL-6 and IL-1 receptor antagonist concentrations in major depression and treatment resistant depression. Cytokine. 1997;9(11):853-8.

77. Sluzewska A, et al. Interleukin-6 serum levels in depressed patients before and after treatment with fluoxetine. Ann N Y Acad Sci. 1995;762:474-6.

78. Costanzo ES, et al. Psychosocial factors and interleukin-6 among women with advanced ovarian cancer. Cancer. 2005;104(2):305-13. 